\title{
On properties of closed/open two-dimensional network-chainmail with different rules of particle movement
}

\author{
Ivan Kuteynikov $^{1,2}$, Marina Yashina ${ }^{1,2}$, Alexander Tatashev ${ }^{1}$ \\ ${ }^{1}$ Department of Higher Mathematics, Moscow Automobile and Road Construction State Technical University (MADI) \\ ${ }^{2}$ Department of Mathematical Cybernetics and Information Technology, Moscow Technical University of Communications and \\ Informatics (MTUCI)
}

\section{Article Info}

Received Dec 15, 2018

\section{Keyword:}

Two-dimensional chainmail

Dynamical systems

Self-organization

Simulation models

\begin{abstract}
With the increase in the number of vehicles and the dimension of road networks, the problem of developing adequate and effective mathematical models to traffic simulation arises. The paper represents the traffic studies based on deterministic two-dimensional network of contours called chainmail introduced by A.P. Buslaev et al. Each contour consists of four cells and one particle moving around it. The open and closed versions of chainmail models with one-directional and co-directional particle movement are considered. The average velocity and other characteristics of chainmail are studied. Four theorems and hypotheses formulated in 2013, 2018 describing the dependence of average velocities of one-directional/co-directional particle movement on initial states of closed/open chainmail are tested and extended with the simulation modeling.
\end{abstract}

\section{Corresponding Author:}

Ivan Kuteynikov,

Department of Mathematical Cybernetics and Information Technology,

Moscow Technical University of Communications and Informatics (MTUCI),

8-a, Aviamotornaya street, Moscow, Russia.

Department of Higher Mathematics,

Moscow Automobile and Road Construction State Technical University (MADI)

64, Leningradsky prospect, Moscow, Russia.

Email: mr.stubbs@rambler.ru

\section{Introduction}

Today, many classic traffic models developed in the mid-20th century are either too simple to simulate traffic on complex networks or the algorithms embedded in their work show unsatisfactory results on large networks with many simultaneously moving objects. One of such classic traffic models is BML (Biham - Middleton Levine) [1]. The underlying cellular automaton, which implements the movement of particles along a square lattice, simulates the movement of vehicles through crossroads. The studies of self-organization (states of free movement from any initial states) of particles and collapse (stop of motion) on the network were provided using computer simulations. Although the model has clear visualization that allows to verify its operation, this model is too simple to model complex network structures of large dimensions.

The works of the team of authors A.P. Buslaev, M.V. Yashina, A.G. Tatashev are devoted to methods of describing flows on various networks. The approaches developed by them describe the traffic model in the form of a discrete dynamic system in which moving particles are used along a system of contours with a network structure. Models of this kind have a limited set of parameters and allow implementation of easy-toread visualization on networks of both large and small dimensions. The restrictions imposed on the network structure and traffic rules allow for an analytical study of systems of this kind. 
In the papers [2], [3] a model consisting of contours forming a regular two-dimensional structure, called a chainmail, is presented. Models of this kind with sets of dynamic rules can be used to predict traffic. For various initial parameters of this model, four theorems and four hypotheses were formulated. Hypotheses were presented without confirmation.

In this paper, the section 2 presents a description of a two-dimensional contour network - chainmail. The rules for movement around the chainmail are presented in the section 3. The section 4 contains characteristics of model such as average velocitie. The results of research including dependence of particle movement, average velocities, number of delays on chainmail dimensions are included in the section 5. For the four hypotheses formulated in [2], [3] for the four theorems, describing established states and average velocities of chainmail for different initial conditions, presented their confirmations and expansions, using the developed computer model. The section 6 summarize the research results and future works.

\section{Two - dimensional contour network - chainmail}

The concept of contour networks was presented in [3] (Kozlov, Buslaev, Tatashev, 2013). This type of network consists of contours - sets of cells combined into subsets with common cells along which particles move in a given direction.

In [5] a closed chain of $N$ contours consisting of four cells was considered (Fig. 1). Each contour has one particle and two adjacent contours with which it has common cell nodes. The chain can be closed or open. Movement along the chain can be both one-directional and co-directional. At simultaneous attempts of particles to occupy a cell common to neighboring contours, the particle of the contour located on the right has the advantage.

The state space of the chain is a vector $\mathrm{X}$, which includes the position of the particle in the contour.

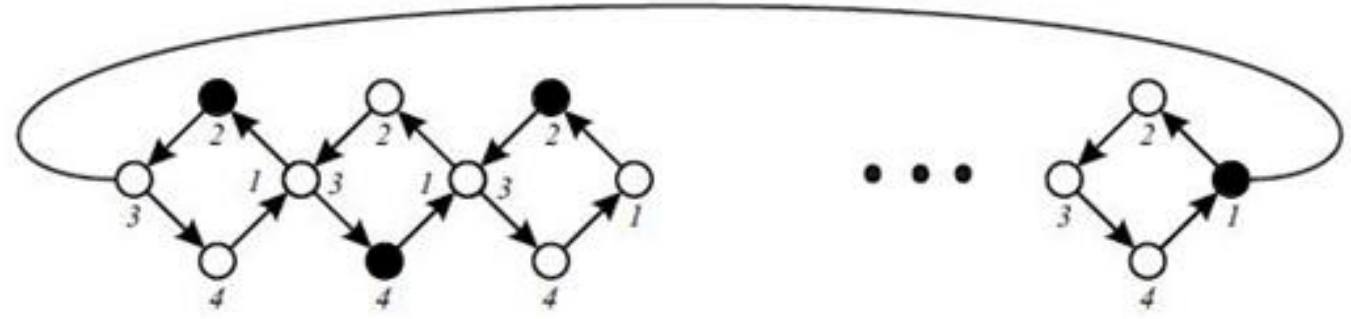

Figure 1. Closed chain of contours with one-directional movement and vector $X=\{2,4,2, \ldots, 1\}$ [2]

In [2],[3],[6] a toroidal system of contours called closed chainmail is represented as a discrete in time and a set of states dynamic system, the carrier of which is a system of $4 m n$ contours of size $2 m x 2 n$, where $\mathrm{m}, \mathrm{n}$, are natural numbers.

On each contour, there are four cells and one particle. Each cell of contour is common for a given contour and one of four adjacent contours. For contour $\mathrm{C}_{\mathrm{i}, \mathrm{j}}$, the adjacent contours are $\mathrm{C}_{\mathrm{i}, \mathrm{j}+1}, \mathrm{C}_{\mathrm{i}, \mathrm{j}-1}, \mathrm{C}_{\mathrm{i}+1, \mathrm{j}}, \mathrm{C}_{\mathrm{i}-1, \mathrm{j}}, i=1, \ldots, 2 m$, $j=1, \ldots, 2 n$.

The cells in the contour are numbered from one in the rightmost cell to four clockwise or counterclockwise. Thus, one cell belonging to two contours will have its own number in each of them.

The state space of the chainmail is a set of vectors $X_{j}$, which includes the position of the particle in the contour $\mathrm{C}_{\mathrm{i}, \mathrm{j}}$.

Open chainmail consists of contours, denoted as $\mathrm{C}_{\mathrm{ij}}$. With $i=1$ contour $\mathrm{C}_{\mathrm{ij}}$ do not have a neighboring contour $\mathrm{C}_{\mathrm{i}+1 \mathrm{j}}$, with $i=2 m$ do not have a neighboring contour $\mathrm{C}_{\mathrm{i}-1 \mathrm{j}}$, with $j=1$ do not have a neighboring contour $\mathrm{C}_{\mathrm{ij}-1}$, with $j=2 n$ do not have a neighboring contour $\mathrm{C}_{\mathrm{ij}+1}$ (Fig.2). 

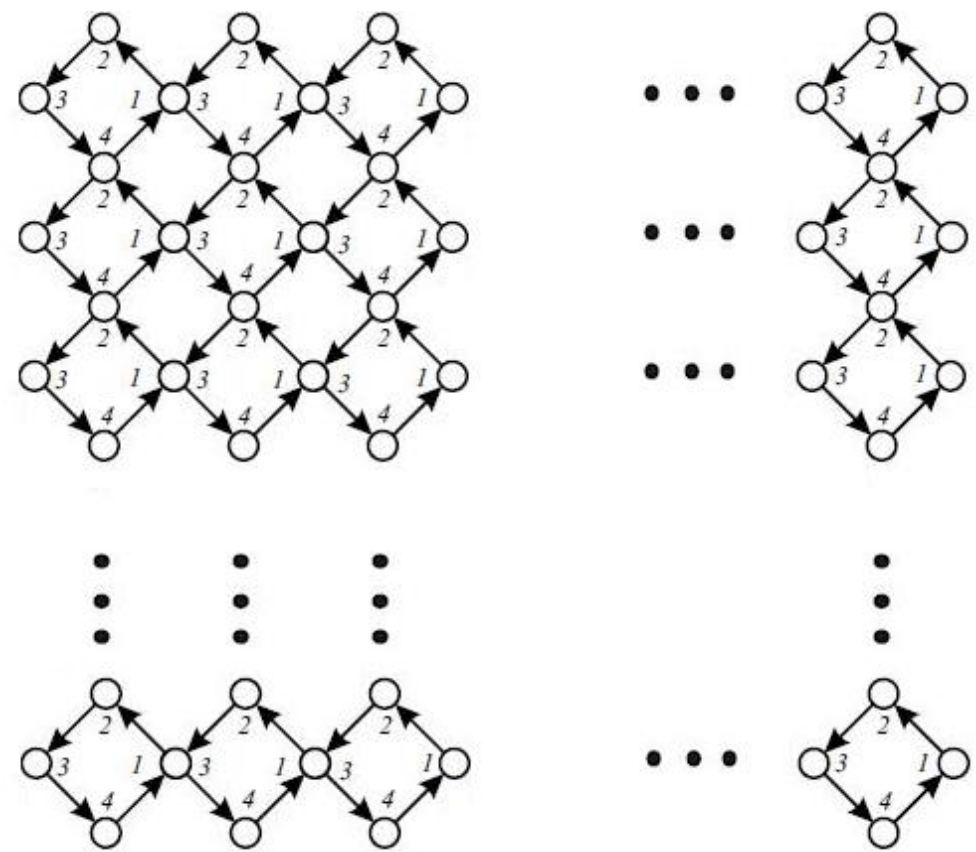

Figure 2. Open chainmail of size $2 m \times 2 n$

\section{Rules of movement on the chainmail}

Chainmail is a system with deterministic particle movement rules. If at the moment of time $t$ the particle is in the cell of the contour with the number $i$, then at the moment of time $t+1$ it will be in the cell with the number $i+1$ (addition modulo 4), $i=1,2,3,4$, if the cell is not occupied. The particle does not move if the cell into which the particle is trying to get is currently occupied by a particle from the neighboring contour. If particles of neighboring contours tend to occupy a common cell at the same time, then only one of these particles will move in accordance with conflict resolution rule. In the case of conflict, the priority is given to the particle with having the least index of $i$ or $j$.

We say that a set of contours is in the state of collapse from time $t 0$ if no particle from this set moves at any time $t \geq t 0$.

The movement of particles along the contour can be one-directional or co-directional.

With one-directional movement, all particles move in the same direction clockwise/counterclockwise (Fig. 3, a).

The co-directional movement consists in the fact that if the particle moves clockwise on the contour, then in the neighboring contours the movement occurs counterclockwise and vice versa (Fig. 3, b).

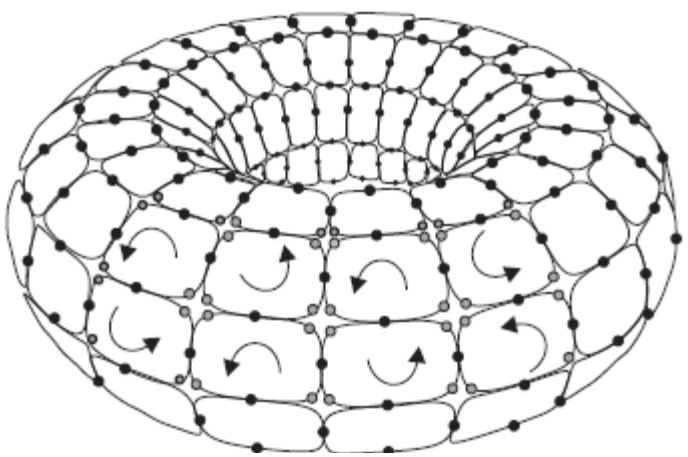

a)

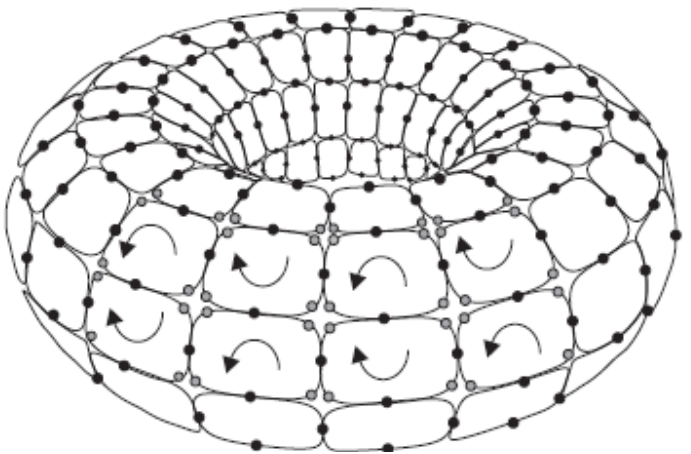

b)

Figure 3. a) Closed chainmail with one-directional movement [3]

b) Closed chainmail with co-directional movement [3] 


\section{Characteristics of chainmail}

The characteristics of the study are the average speed of movement along the chainmail $V_{a}(t)=P / 4 m n$, where $P$ - number of particles, moved during current iteration $t$, as well as the number of delays in the movement of particles - $D$.

Let $k$ be the greatest divisor of $m$ and $n$. The set of contours can be divided into $k$ subsets such that, if one of the contours of this subset is in a state of collapse, then the subset is in a state of collapse. Such sets are called diagonals (Figure 4). Each diagonal contains the same number of contours. Since there are $4 m n$ contours in the chainmail, then each diagonal contains $4 m n / k$ contours.
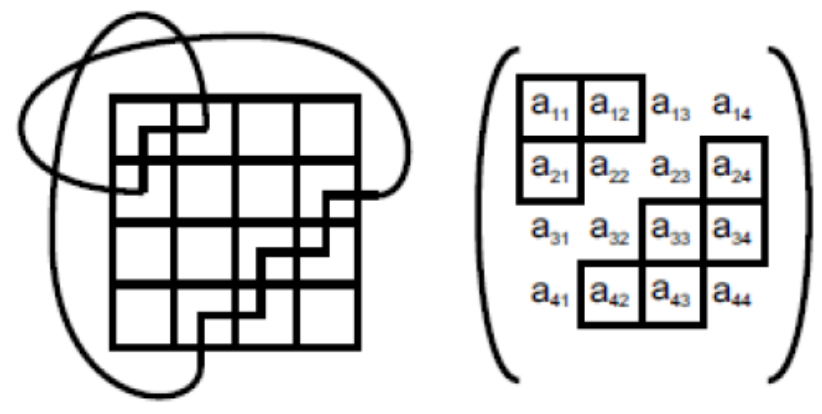

Figure 4. Diagonal on closed $4 \times 4$ chainmail [3]

The established regime of movement $T$ will be considered as the number of iterations since when $\mid \mathrm{V}(\mathrm{t}+1)$ $\mathrm{V}(\mathrm{t}) \mid<\varepsilon$ for $k$ and more iterations, there $\varepsilon$ is an accuracy.

\section{Simulation of the particle movement along the chainmail}

For computer simulation the software package in C\# programming language was developed. Using it, the movement of particles on chainmail was simulated in accordance with the initial conditions presented in the theorems described in [2], [3]. Theorem 1 describes the dependence of velocities of particles on initial states of closed chainmail with co-directional movement. Availability of free movement for open chainmail with codirectional movement is described in Theorem 2. The appearance of sets of contours and the dependence of their velocities on the initial states for closed chainmail with one-directional movement is described in Theorem 3. Theorem 4 describes the states of free movement and collapse for sets of contours for open chainmail with one-directional movement.

For these theorems, a computer simulation method was used to test the formulated hypotheses and analyze the dependence of average velocities and number of delays on chainmail dimension.

\subsection{Closed chainmail with co-directional movement}

Theorem 1 [3]. For any closed chainmail with co-directional movement, the following is true. For any set of integer numbers $s_{1}, \ldots, s_{k}, 0 \leq s_{i} \leq m n / k$, there exists an initial state such the velocity of particles on each contour of the $i_{\text {th }}$ diagonal is equal to $s_{i} k /(m n), i=1, \ldots, k$.

Hypothesis 1 [3]. For any spectral cycle of closed chainmail with co-directional movement, average velocities are such as it is described in condition of Theorem 1.

As a result of multiple simulations, Hypothesis 1 is confirmed. The average particle velocity on each $i_{t h}$ diagonal takes one of the values $\operatorname{sik} /(m n), i=1, \ldots, k$. (Figure 6). 


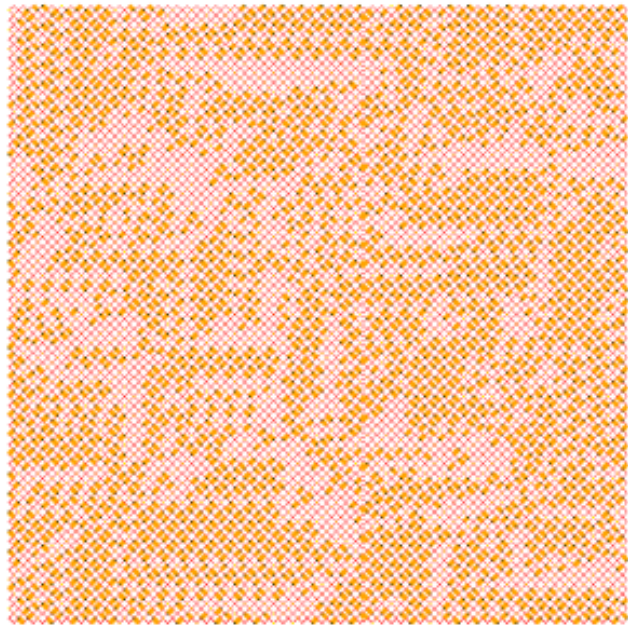

a)

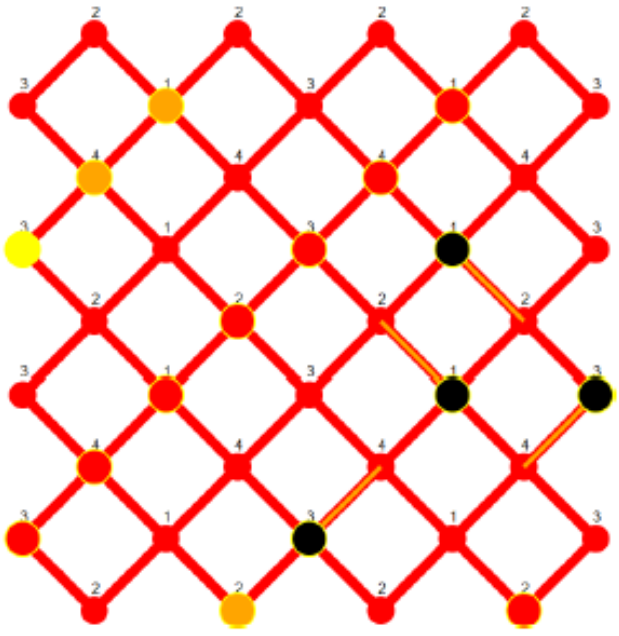

b)

Figure 5. a) Co-directional movement along a closed chainmail of size $64 \times 64$

b) Co-directional movement along a closed chainmail of size $4 \times 4$. There are four particle colors: black moved on previous iteration, yellow - have not moved for 1 iteration, orange - haven't moved for 2 iterations, red - haven't moved for more than 3 iterations

The established regime of movement for $\varepsilon=0.0001$ is $\mathrm{T}_{16 \times 16}=57, \mathrm{~T}_{32 \times 32}=60, \mathrm{~T}_{64 \times 64}=107, \mathrm{~T}_{128 \times 128}=115$, $\mathrm{T}_{512 \times 512}=86$.

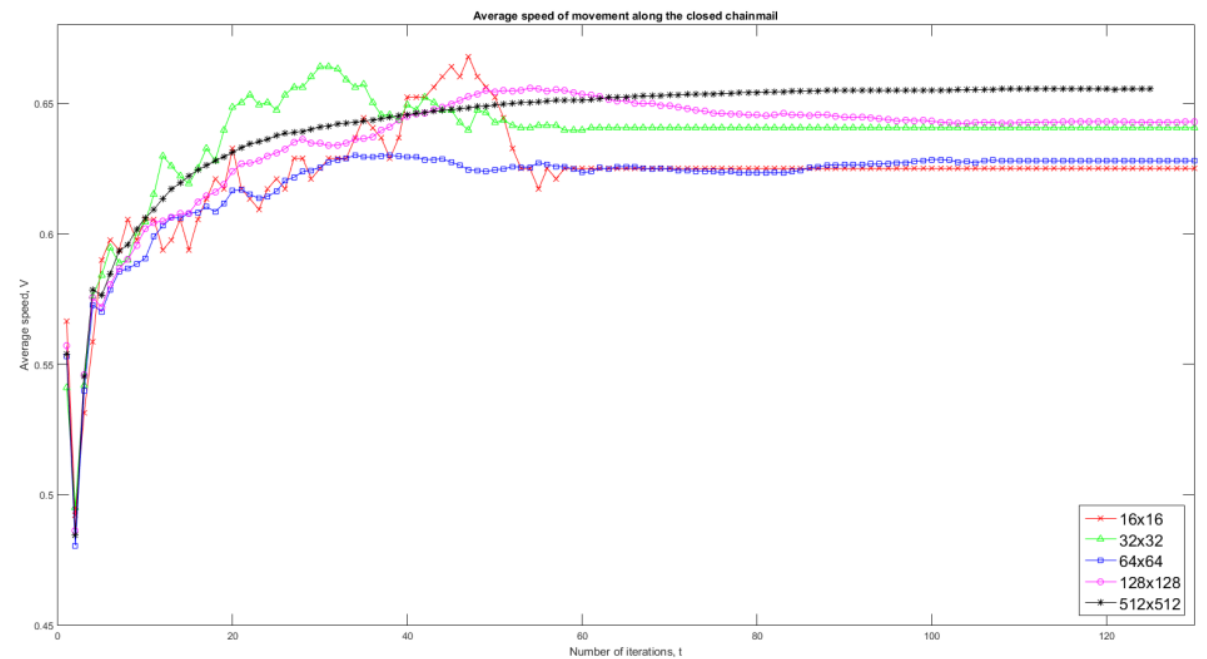

Figure 6. Dependence of the average speed along a closed chainmail of sizes $16 \times 16,32 \times 32,64 \times 64,128 \times 128$, $512 \times 512$ on the number of iterations 


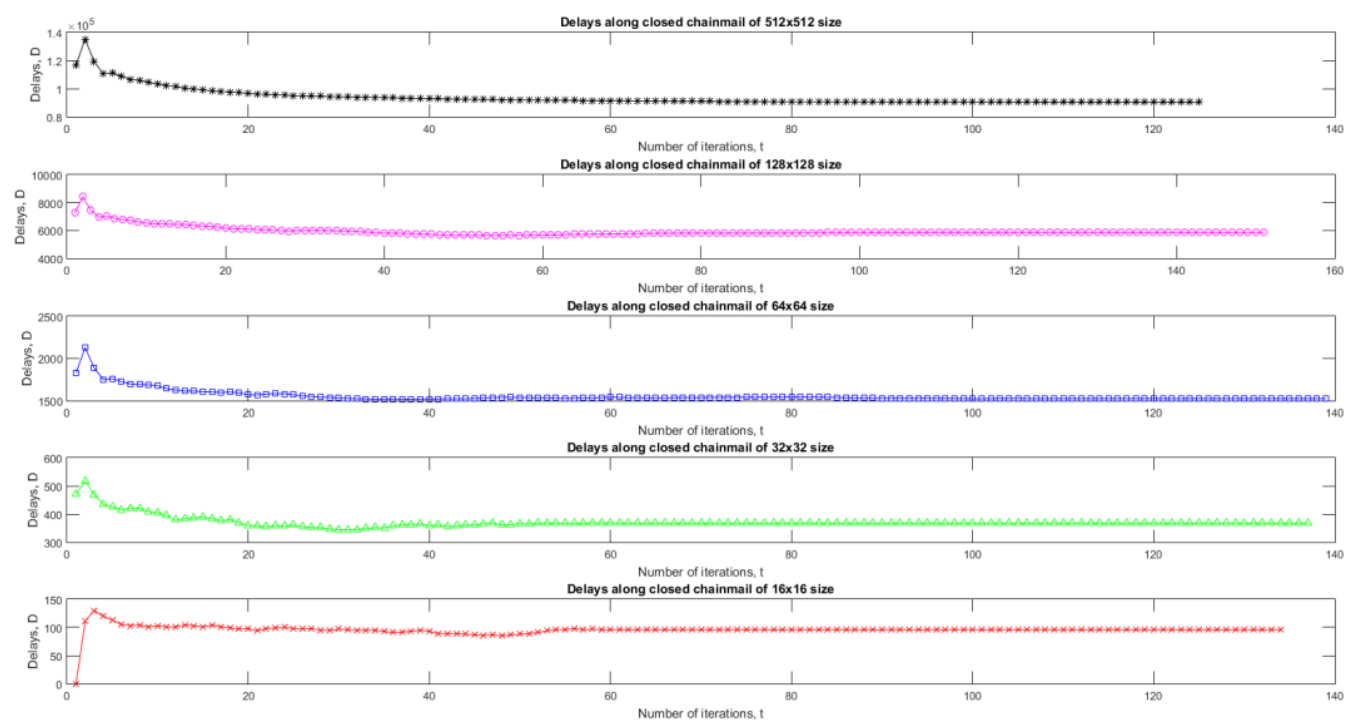

Figure 7. Dependence of the number of delays along a closed chainmail of sizes $16 \times 16,32 \times 32,64 \times 64$, $128 \times 128,512 \times 512$ on the number of iterations

\subsection{Open chainmail with co-directional movement}

Theorem 2 [2]. For an open chainmail with co-directional movement, there are states of free movement.

Hypothesis 2 [2]. Open chainmail with co-dimensional movement has the property of self-organization, i.e., the systems comes to the state of free movement from any initial state (all particles move at every step from some moment).

As a result of multiple simulations, Hypothesis 2 is confirmed. Before reaching the number of iterations equal to the length of the diagonal, the diagonals are shifted along their path, after which the system establishes an state of free movement (Fig. 8) with an average speed of 1 (Fig. 9) and zero conflicts (Fig. 10).

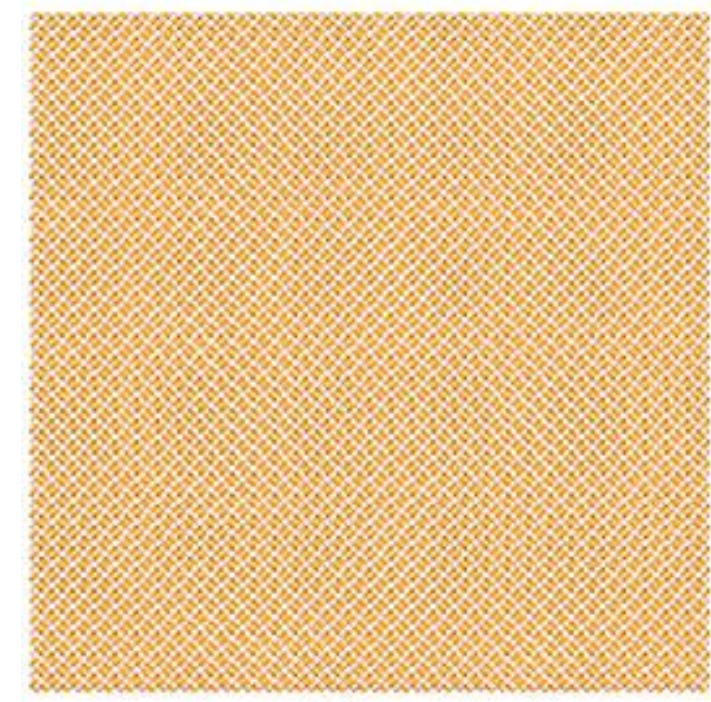

a)

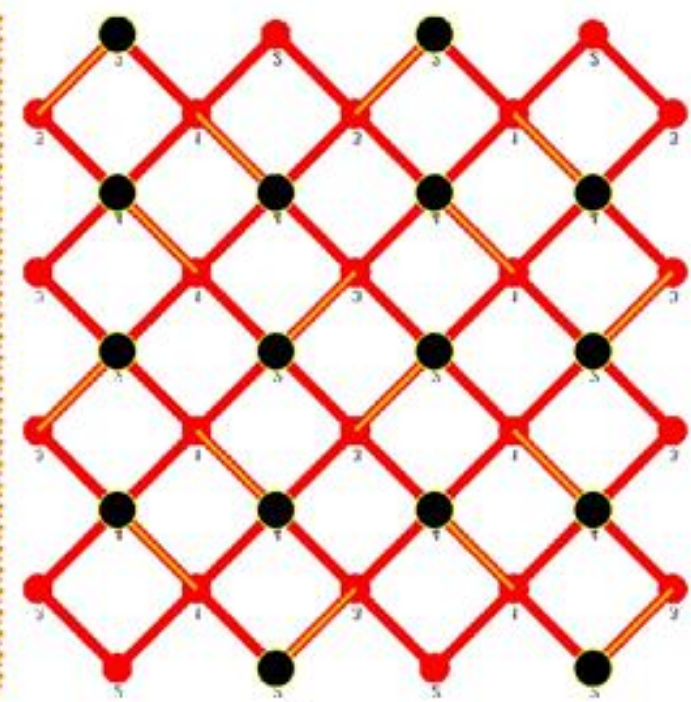

b)

Figure 8. a) The established state of free movement along open chainmail of size $64 \times 64$

b) Co-directional movement along an opened chainmail of size $4 \times 4$

The established regime of movement for $\varepsilon=0.0001$ is $\mathrm{T}_{16 \times 16}=36, \mathrm{~T}_{32 \times 32}=75, \mathrm{~T}_{64 \times 64}=142, \mathrm{~T}_{128 \times 128}=274$, $\mathrm{T}_{512 \times 512}=1036$. It can be seen that the established regime of movement occurs after reaching a number of iterations equal to the length of the chainmail diagonal. 


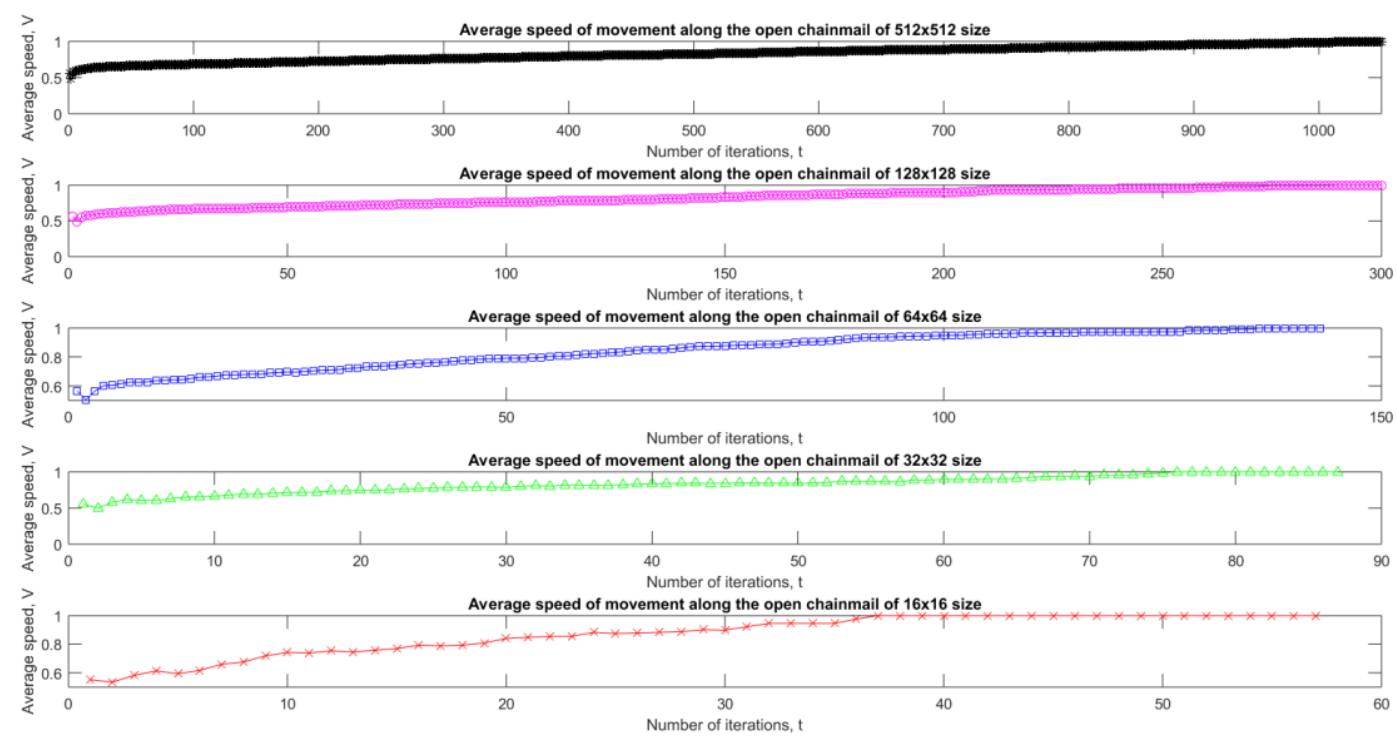

Figure 9. Dependence of the average speed along an open chainmail of sizes $16 \times 16,32 \times 32,64 \times 64,128 \times 128$, $512 \times 512$ on the number of iterations
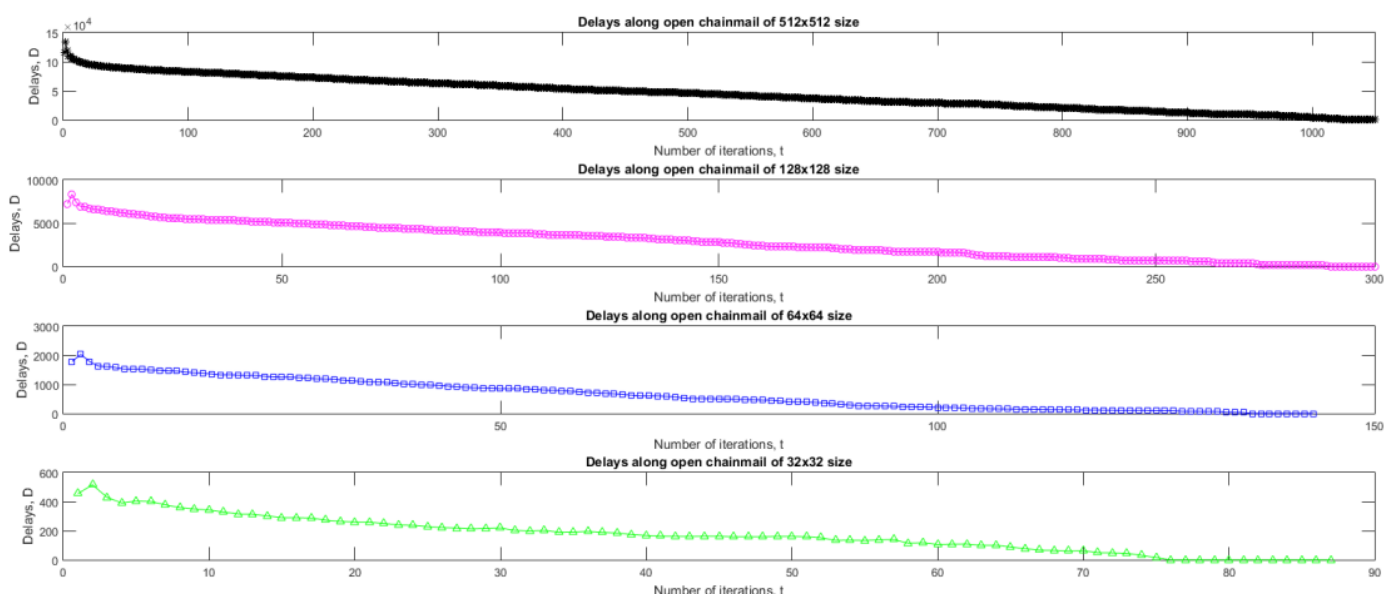
Delays along open chainmail of $16 \times 16$ size

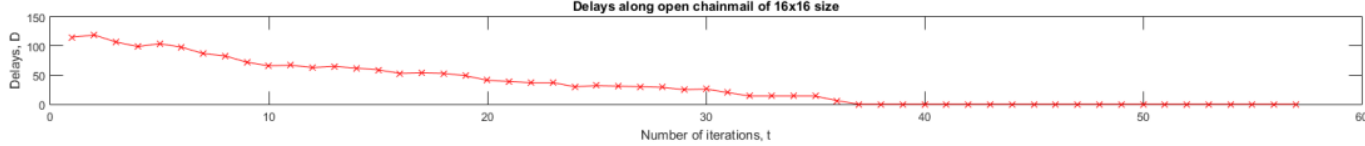

Figure 10. Dependence of the number of delays along an open chainmail of sizes $16 \times 16,32 \times 32,64 \times 64$, $128 \times 128,512 \times 512$ on the number of iterations

\subsection{Closed chainmail with one-directional movement}

Theorem 3 [3]. The following statements are true for a closed chainmail with one-directional movement.

1) Suppose the number $k$ and $i_{1}, \ldots, i_{k}, j_{1}, \ldots, j_{k}$, satisfy the following conditions. The set of all contours is divided into $k$ sets $G_{i j j}, \ldots, G_{i k j k}$ and complement $G$ of union of $G_{i l j} 1, \ldots, G_{i k j k}$. Then there exists an initial state such that sets $G_{i l j l}, \ldots, G_{i k j k}$ are in the state of collapse, and every particle of the set $G$ moves at each step.

2) For any number $s=0,1, \ldots,[n / 3]$ there exist initial states of the system such that the average velocity of the particle of each contour is equal to $(n-s) / n$.

3) For any number $s=0,1, \ldots,[\mathrm{m} / 3]$ there exist initial states of the system such that the average velocity of the particle of each contour is equal to $(m-s) / m$.

Hypothesis 3 [3]. Any spectral cycle of a closed chainmail with one-directional movement satisfies the condition of Theorem 3. 
As a result of multiple simulations, Hypothesis 3 is confirmed. In the process of movement, blocks of size $2 \times 2$ are formed from the contours (Figure 11). Depending on the selected contour $\mathrm{C}_{\mathrm{ij}}$, there are four possible block types: $\left\{\mathrm{C}_{\mathrm{ij}}, \mathrm{C}_{\mathrm{ij}+1}, \mathrm{C}_{\mathrm{i}+1 \mathrm{j}}, \mathrm{C}_{\mathrm{i}+1 \mathrm{j}+1}\right\},\left\{\mathrm{C}_{\mathrm{ij}}, \mathrm{C}_{\mathrm{ij}-1}, \mathrm{C}_{\mathrm{i}+1 \mathrm{j}}, \mathrm{C}_{\mathrm{i}+1 \mathrm{j}-1}\right\},\left\{\mathrm{C}_{\mathrm{ij}}, \mathrm{C}_{\mathrm{ij}+1}, \mathrm{C}_{\mathrm{i}+1 \mathrm{j}}, \mathrm{C}_{\mathrm{i}-1 \mathrm{j}+1}\right\},\left\{\mathrm{C}_{\mathrm{ij}}, \mathrm{C}_{\mathrm{ij}-1}, \mathrm{C}_{\mathrm{i}-1 \mathrm{j}}, \mathrm{C}_{\mathrm{i}-1 \mathrm{j}-1}\right\}$ $i=1, \ldots, 2 m, j=1, \ldots, 2 n$.

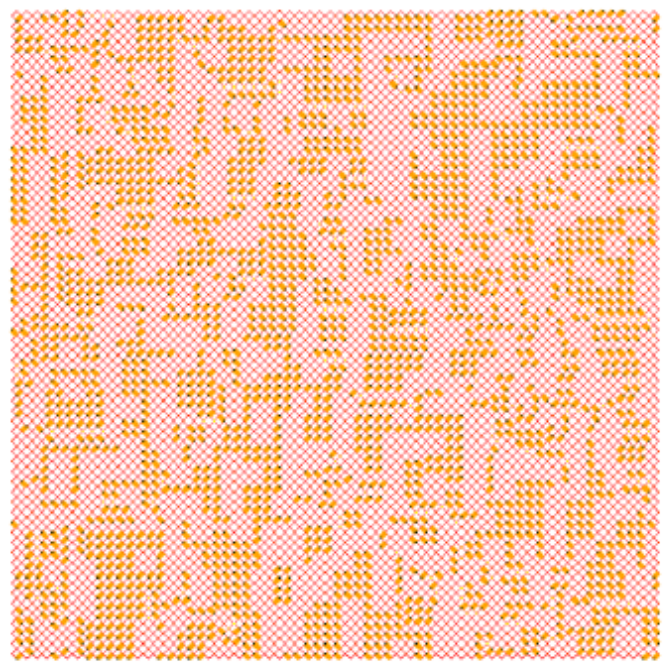

a)

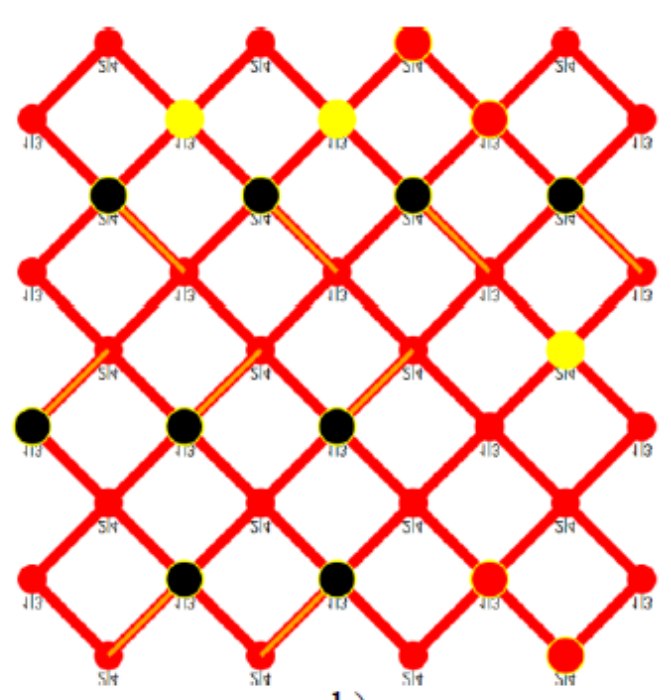

b)

Figure 11: a) One-directional movement along a closed chainmail of size $64 \times 64$ b) One-directional movement along a closed chainmail of size $4 \times 4$

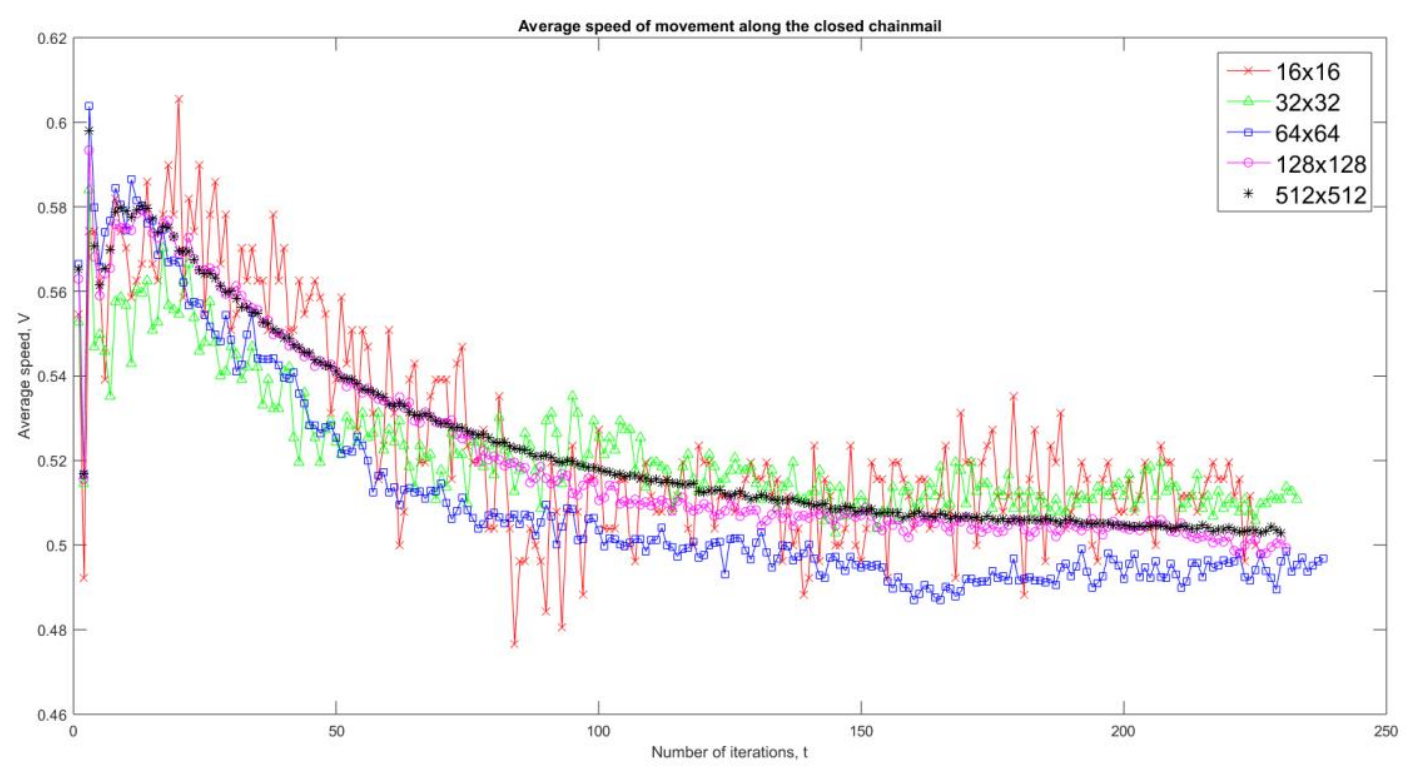

Figure 12. Dependence of the average speed along a closed chainmail of sizes $16 \times 16,32 \times 32,64 \times 64,128 \times 128$, $512 \times 512$ on the number of iterations

To find the established regime of movement for one-directional movement, an approximation of the mean velocity values is required. 


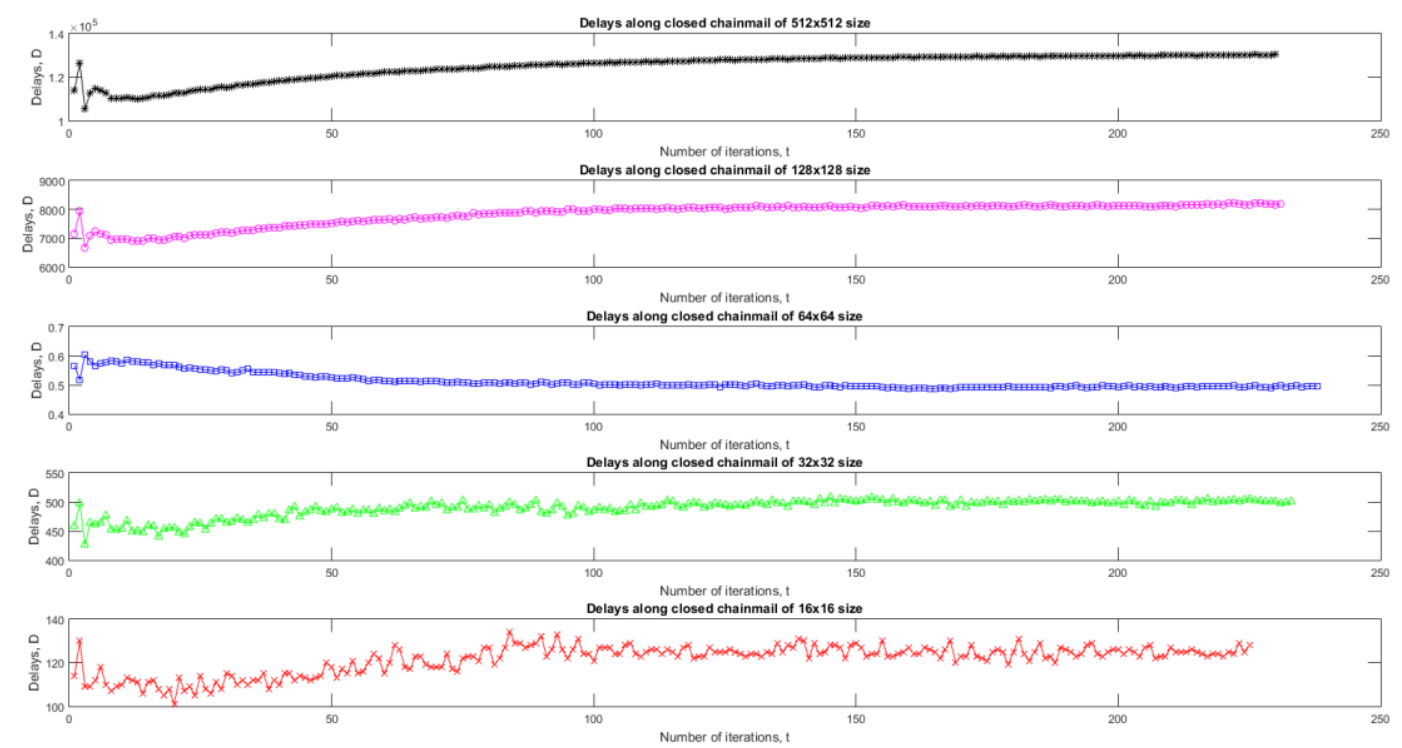

Figure 13. Dependence of the number of delays along an open chainmail of sizes $16 \times 16,32 \times 32,64 \times 64$, $128 \times 128,512 \times 512$ on the number of iterations

On the Figures of the average speed versus the number of iterations (Figure 12) and the number of delays on the iterations (Figure 13), it is clear that the system ultimately comes to an equilibrium state.

\subsection{Open chainmail with one-directional movement}

Theorem 4 [2]. The following statement is true for an open chainmail with one-directional movement. Assume that the number $k$ and indexes $i_{1}, \ldots, i_{k}, j_{1}, \ldots, j_{k}$, satisfy the following. The set of all contours is divided into $k$ sets of $G_{i l j}, \ldots, G_{i k j k}$ and complement $G$ of union of $G_{i l j l}, \ldots, G_{i k j k}$. Then there exists an initial state such that sets of $G_{i l j l}, \ldots, G_{i k j k}$ are in the state of collapse, and every particle of the set $G$ moves at each step.

Hypothesis 4 [2]. Any spectral cycle of an open chainmail with one-directional movement corresponds to the first satisfies the condition of Theorem 4.

As a result of multiple simulations, Hypothesis 4 is confirmed. There is a slight increase in the average speed, due to the fact that the nodes of the contours along the perimeter of the open chainmail do not participate in conflicts. With an increase in the size of the chainmail, the effect decreases (Fig. 15).

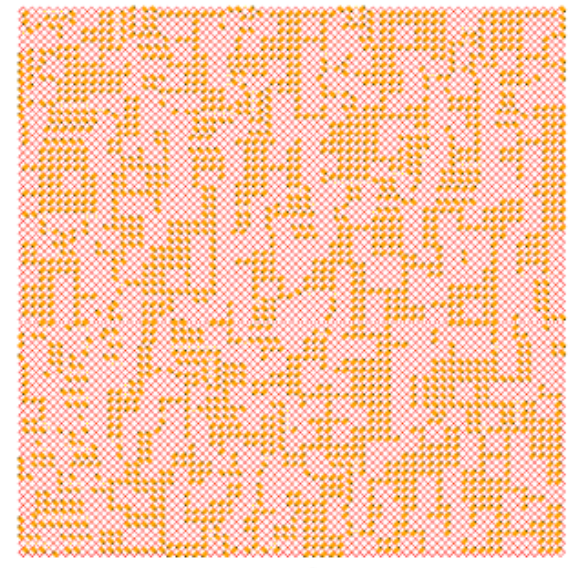

a)

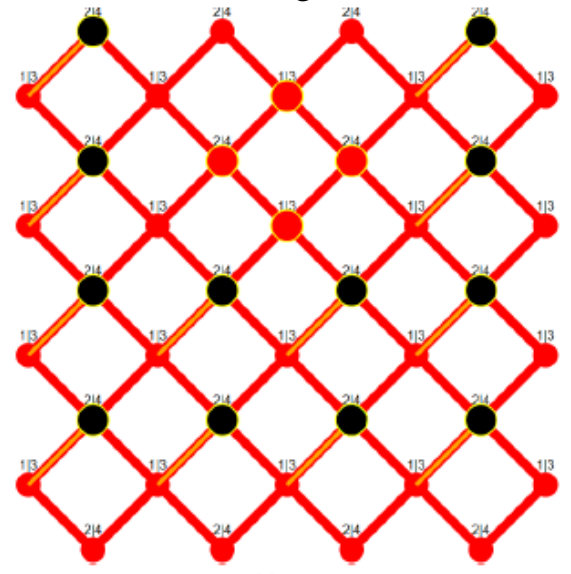

b)

Figure 14: a) One-directional movement along an open chainmail with size $64 \times 64$

b) One-directional movement along an open chainmail with size $64 \times 64$ 


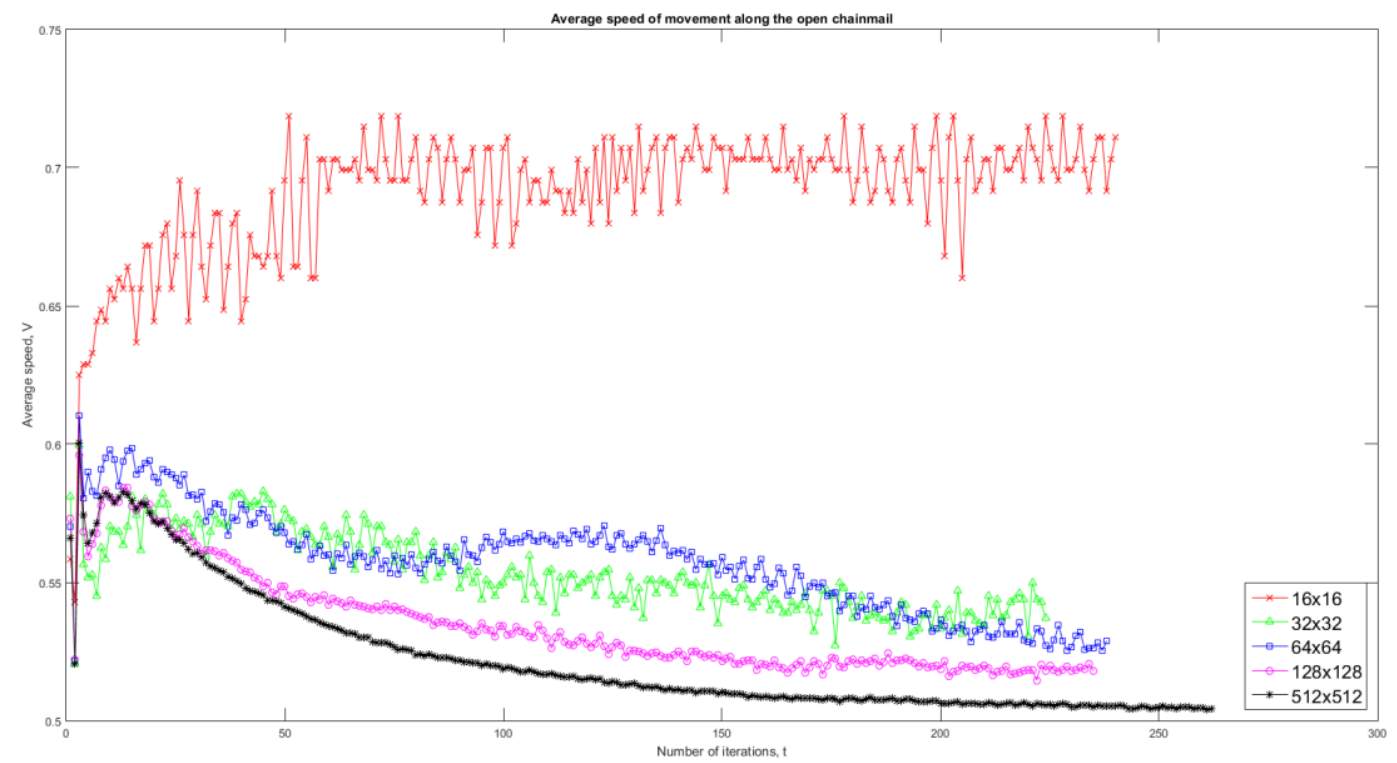

Figure 15. Dependence of the average speed along an open chainmail of sizes $16 \times 16,32 \times 32,64 \times 64,128 \times 128$, $512 \times 512$ on the number of iterations
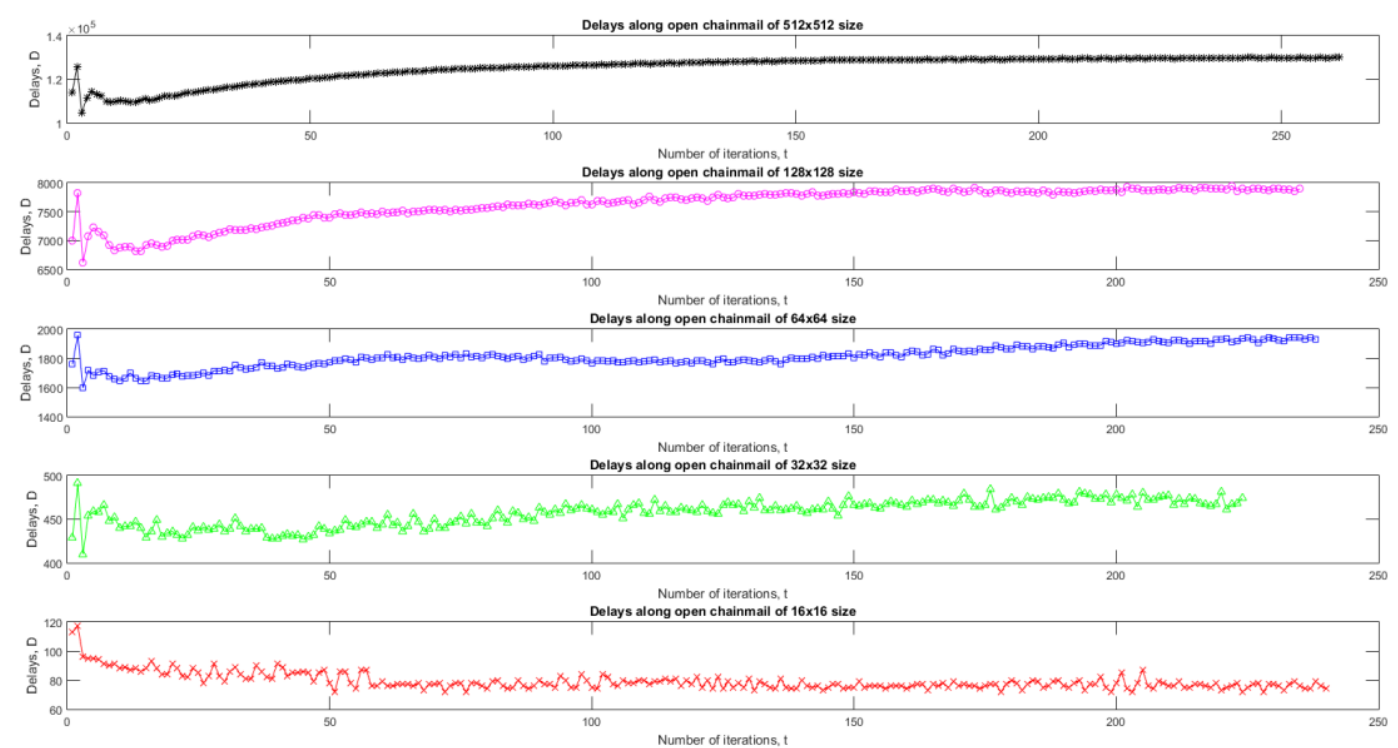

Figure 16. Dependence of the number of delays along an open chainmail of sizes $16 \times 16,32 \times 32,64 \times 64$, $128 \times 128,512 \times 512$ on the number of iterations

\section{Conclusions}

As a result of the simulation, all four hypotheses were confirmed. It has been observed that, according to the law of large numbers, chainmails of larger dimension give a smaller scatter when calculating the average velocity.

The future works will include:

- research of the influence of different priorities rules of particle movement in conflicts resolution;

- selection of diagonals on the chainmail obtaining their length, speed and spectral cycle;

- research of chainmails with contours including more than four cells in each of them. 


\section{Acknowledgements}

This work has been supported by Russian Foundation for Basic Research Grant No. 17-01-00821-a and grant No. 17-29-03419-ofi-m.

\section{References}

[1] Biham O., Middleton A. A., Levine D. Self-organization and a dynamical transition in traffic-flow models //Physical Review A. - 1992. - T. 46. - №. 10. - C. R6124.

[2] Buslaev, A. P., A. G. Tatashev, and M. V. Yashina. "Qualitative properties of dynamical system on toroidal chainmail." AIP Conference Proceedings. Vol. 1558. No. 1. AIP, 2013.

[3] Fomina, Maria Ju, et al. "Cellular Automata as Traffic Models and Spectrum of Two-Dimensional Contour Networks-Open Chainmails." 2018 IEEE International Conference" Quality Management, Transport and Information Security, Information Technologies"(IT\&QM\&IS). IEEE, 2018.

[4] Kozlov, V. V., A. P. Buslaev, and A. G. Tatashev. "On synergy of totally connected flows on chainmails." Proc. of the 13 International Conference on Computational and Mathematical Methods in Science and Engineering, Almeria, Spain. Vol. 3. 2013.

[5] Kozlov, Valery V., Alexander P. Buslaev, and Alexander G. Tatashev. "Behavior of pendulums on a regular polygon." Journal of Communication and Computer 11 (2014): 30-38. 